\title{
Interactive comment on "Effects of Coupling a Stochastic Convective Parameterization with Zhang-McFarlane Scheme on Precipitation Simulation in the DOE E3SMv1 Atmosphere Model' by Yong Wang et al.
}

Yong Wang et al.

yaokgwy@163.com

Received and published: 28 January 2021

The response was uploaded in the form of a supplement

Please also note the supplement to this comment:

https://gmd.copernicus.org/preprints/gmd-2020-249/gmd-2020-249-AC1- 
2020.

GMDD

Interactive

comment 